\section{An Intelligent Rule-based System for Status Epilepticus Prognostication}

\author{
Bahare Danaei $^{1 \oplus}$, Reza Javidan², Maryam Poursadegh- \\ fard $^{3 * 0}$, Mohtaram Nematollahi ${ }^{4}$
}

\begin{abstract}
Background: Status epilepticus is one of the most common emergency neurological conditions with high morbidity and mortality.

Objective: The aim of this study is to propose an intelligent approach to determine prognosis and the most common causes and outcomes based on clinical symptoms.
\end{abstract}

Material and Methods: In this descriptive-analytic study, a perceptron artificial neural network was used to predict the outcome of patients with status epilepticus on discharge. But this method, which is understandable, is known as black boxes. Therefore, some rules were extracted from it in this study. The case study of this paper is data of Nemazee hospital patients.

Results: The proposed model was prognosticated with $70 \%$ accuracy, while Bayesian network and Random Forest approaches have 51\% and 46\% accuracy. According to the results, recovery and mortality groups had often used phenytoin and anesthetic drugs as seizure controlling drug, respectively. Moreover, drug withdrawal and cerebral infarction were known as the most common etiology for recovery and mortality groups, respectively and there was a relationship between age and outcome, like in previous studies.

Conclusion: To identify some factors affecting the outcome such as withdrawal, their effects either can be avoided or can use sensitive treatment for patients with poor prognosis.

Citation: Danaei B, Javidan R, Poursadeghfard M, Nematollahi M. An Intelligent Rule-based System for Status Epilepticus Prognostication. $J$ Biomed Phys Eng. 2021;11(2):185-196. doi: 10.31661/jbpe.v0i0.916.

\section{Keywords}

Intelligent Approaches; Data Mining; Artificial Neural Networks; Rule Based Systems; Status Epilepticus; Prognosis

\section{Introduction}

tatus epilepticus (SE) is a neurologic disorder with high mortalS ity and morbidity; defined as a long time and non-stopping seizure, or two or more discrete seizures without complete recovery of consciousness between them $[1,2]$. Some studies have reported that the duration of seizure lasts 20-30 minutes, but others have said more than 5 minutes [3]. The risk of this clinical situation is its long attacks since experiments on the brains of adult monkeys have indicated that a continuous attack lasting for about 45 to 60 minutes is sufficient to damage the nerve cells [4]. Owing to high mortality and morbidity of SE patients, there is an essential need to intelligent approaches to determine the prognosis of these patients at the time of discharge, one of
${ }^{1} \mathrm{MSc}$, Department of

Computer Engineering

and Information Technol-

ogy, Shiraz University of

Technology, Shiraz, Iran

${ }^{2} \mathrm{PhD}$, Department of

Computer Engineering

and Information Technol-

ogy, Shiraz University of

Technology, Shiraz, Iran

${ }^{3} \mathrm{MD}$, Clinical Neurology

Research Center, Shiraz

University of Medical Sci-

ences, Shiraz, Iran

${ }^{4} \mathrm{PhD}$, Department of

Health Information Man-

agement, Shiraz Univer-

sity of Medical Sciences,

Shiraz, Iran

*Corresponding author:

Maryam Poursadeghfard

Clinical Neurology

Research Center, Shiraz

University of Medical Sci-

ences, Shiraz, Iran

E-mail: Poursadra@

gmail.com

Received: 24 February 2018 Accepted: 14 April 2018 
which is data mining. Data mining is an analyzing process of the data obtained from different perspectives by summarizing it to new useful information. On the other hand, in spite of the fact that it is a way with the least user intervention, it is able to express the logical relations between data [5]. One of the most accurate and reliable techniques of data mining is Artificial Neural Network (ANN) which solves the problems with no algorithmic solution or very complex algorithmic solution. Despite its high accuracy, ANN is known as a black box and can't express and interpret the behavior of the model and the reason of prediction [6]. In recent years, many studies have been carried out on rule extracting from ANN [6]. They first selected the appropriate structure for the network and trained it and then pruned the created model to reduce the connections and neurons. Next, the outputs of the hidden layer are discretized in order to be extracted from them and finally pruned and generalized the specific rules. The use of ANN in neurology includes analysis of Electroencephalography (EEG) signals test for seizure detection because the evaluation of these signals is very time-consuming and tedious. [4, $7,8]$. We found a study about SE prognosis based on the clinical symptoms by using intelligent approaches that it evaluate the underlying etiologic factors of epilepsy patients and predict the prognosis of these patients by using a Multi-Layer Perceptron Neural Network (MLPNN) based on risk factors. The results showed that the most important risk factor of epilepsy was related to some items, including the febrile seizure, the parents' kinship, the history of epilepsy in relatives and the history of head trauma. The correct prediction rate for detection of the prognosis was $91.1 \%$ by using the MLPNN algorithm [9]. Moreover, there are some studies based on statistical methods [10-13]. In these statistical studies, the frequency of symptoms for each group has only been introduced, and none of them are based on prognostication.
However, many studies were carried out about SE in Iran, particularly in pediatric patients [14-17], there is a gap for adults [10, 18] The proposed method in this paper provides concise rules instead of existing weights in network structure, which is easily checked by an expert, and finally presents a new perspective of the data to system users. In fact, this study aims to determine the prognosis for adult patients with SE and the most important causes of the seizures via interpretable ANN, through symptoms without results of the EEG or Magnetic Resonance Imaging (MRI) test like some other studies [13].

The proposed method has been tested by some well-known UCI Machine Learning Repository datasets. Therefore, an application was proposed to manage SE patients data and determine the prognosis of their outcome on discharge time with ANN approach in this study. Next, we generated a set of rules similar to the approach proposed by Kamruzzaman et al., [6].

\section{Material and Methods}

In this descriptive-analytic study, the major steps of the proposed method are summarized in Figure 1 and explained further in subsec-

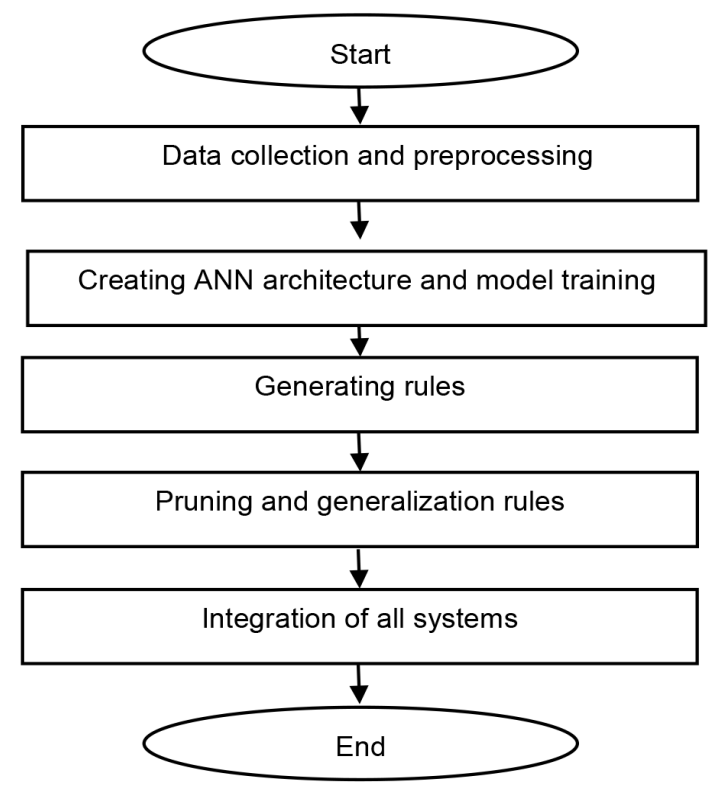

Figure 1: Flowchart of the proposed method 
tions.

\section{Data collection and preprocessing}

The subjects of this study were the adult patients with SE (either convulsive or nonconvulsive) who were admitted in Nemazee hospital (Shiraz, south of Iran) during January 2006 to February 2012. This research is carried out on patients' records, retrospectively. The data were recorded after admission of SE patients, during the hospital course by a daily questionnaire. The patients may be discharged or died by now. The medical research ethics committee of Shiraz University of Medical Science approved the study protocol (approval number: 55-4082). The duration of seizure in these patients was at least 20 minutes or at least two seizure attacks without returning to normal level of consciousness between them for convulsive SE (the definition of SE at that time); furthermore, clinically unrecognized seizure by evidence of SE in EEG for nonconvulsive SE. These data include 134 records and 12 fields including gender, age, duration of epilepsy, the cause of the epilepsy, seizure occurrence in the last six months, prior medication, status type, etiology, course of disease, seizure-controlling drugs, duration of the hospitalization and patients outcome on discharge with four values of mortality, severe disability, moderate disability and good recovery. The patient's outcome on discharge was based on Glasgow Outcome Scale (GOS) which is a scale to divide patients with brain injuries into five groups such as death, persistent vegetative state, severe disability, moderate disability and low disability [19]. However, owing to the low count of patients in group 2, this group was merged with group 3. Indeed, patients were classified into three age groups, including 19-39, 40-59, and $\geq 60$ years. Although, 134 instances are insufficient for data mining techniques, we wanted the results be based on the real statistics in Iran. Thus, we didn't simulate instances. Table 1 shows the collection of the attributes with a set of the possible values.

In order to use the $\mathrm{ANN}$, all data must be converted from nominal status to numerical status. Therefore, each value of each field is converted to a number. Then all the values were normal in the interval $[0,1]$ through Min-Max method as described in Equation (1), since the ANN training is very sensitive

Table 1: The collection of the data and values

\begin{tabular}{cc} 
Attribute Name & Values \\
\hline Gender & Male, Female \\
\hline Age, (years) & $(19-39),(40-59,(60$ and more $)$ \\
\hline Duration of Epilepsy (years) & Secondary, Idiopathic, Unknown \\
\hline Cause of Epilepsy & Yes, No \\
\hline Seizures in last 6 months & Carbamazepine, Sodium Valproate, Phenytoin, Lamotrigine, Phenobarbital, Topir-amate \\
\hline Prior Medication & Convulsive, Myoclonus, Non Convulsive \\
\hline Status Epilepticus Type & Medication Withdrawal, Metabolic Abnormalities, Tumor, Brain Infection, Trauma, Hypoxic, Cere- \\
\hline Etiology & bral Infarction, Cerebral Venous Thrombosis, Drug/Substance Abuse, Multiple Sclerosis \\
\hline Course of Disease & Acute, Non Acute \\
\hline Seizure-Controlling Drugs & Phenytoin, Depakin, Phenobarbital, Anesthetic, Others \\
\hline Duration of Hospitalization & (2-100) days \\
\hline Glasgow Outcome Scale (GOS) & Mortality, Severe Disability, Moderate Disability, Good Recovery
\end{tabular}


and the mapping of inputs to outputs is totally dependent on the inputs.

Normalize $(X)=\frac{X-\min (X)}{\max (X)-\min (X)} \times($ NewMax - NewMin $)+$ NewMin (

Where $\min (x)$ and $\max (x)$ are the minimum and maximum values for $x$, respectively; and here, NewMax and NewMin are 0 and 1, respectively.

Creating ANN architecture and model training

In this study, in order to create the ANN model, the Neural Network toolbox from MATLAB R2011a was used. Thus a 2-layer perceptron network was used, including 11 inputs and 1 output neuron. To determine the number of hidden neurons and the training and transfer functions, the trial and error methods were used. As a result, the performance of the model for different settings was evaluated through Mean Squared Error (MSE) performance function which is available in Equation (2):

$$
M S E=\frac{1}{N} \sum_{i=1}^{N}\left(t_{i}-o_{i}\right)^{2}
$$

Where $N$ is the total number of examples, $t_{i}$ is the desired output and $o_{i}$ is the actual output of the network. Therefore, the performance of the model was calculated by $4,5,6, \ldots, 10$ neurons in the hidden layer. Owing to a lot of tests, choosing hidden neurons of 4 changes to
10; the training network, with fewer than four hidden neurons, was not carried out properly; on the other hand, because of the low number of training samples, the network should not be larger than this. Moreover, data was divided into three portions, including $80 \%$ of data for training, $10 \%$ for validation and $10 \%$ for the test model. In order to have more reliability, 10 -Fold cross validation was also used. Therefore, the data set was divided into ten subsets, and the training was repeated ten times. Each time, one of the ten subsets was used as the test set, and one of the other nine subsets was used as the validation set and the other eight subsets were used as the training set. Then the average MSE of all ten trials was computed.

The performance of the model with tansig and logsig transfer functions for hidden layers and traingdm, trainscg, trainlm, trainbr and trainrp training functions was calculated. Thus the results of the 700 iterations of the training are available in Table 2 and lead to using eight hidden neurons, the trainscg training method, tansig transfer function for hidden layers and linear transfer functions for output layer had the best performance and the least MSE equal to 0.0499 .

\section{Generating rules}

There are different techniques for rule extraction from ANN; one of which is decomposition method, including analysis of each

Table 2: The performance evaluation of the 700 iterations of the training

\begin{tabular}{|c|c|c|c|c|c|c|c|c|c|c|c|}
\hline \multirow{2}{*}{\multicolumn{2}{|c|}{$\begin{array}{l}\text { Training Function } \\
\text { Transfer Function }\end{array}$}} & \multicolumn{2}{|c|}{ Trainrp } & \multicolumn{2}{|c|}{ Trainbr } & \multicolumn{2}{|c|}{ Trainlm } & \multicolumn{2}{|c|}{ Trainscg } & \multicolumn{2}{|c|}{ Traingdm } \\
\hline & & Tansig & Logsig & Tansig & Logsig & Tansig & Logsig & Tansig & Logsig & Tansig & Logsig \\
\hline \multirow{7}{*}{$\begin{array}{c}\text { The number } \\
\text { of hidden } \\
\text { neurons }\end{array}$} & 4 & 0.0975 & 0.098 & 0.0844 & 0.0841 & 0.1403 & 0.1138 & 0.0948 & 0.098 & 0.1877 & 0.1055 \\
\hline & 5 & 0.1132 & 0.0969 & 0.0844 & 0.0841 & 0.1361 & 0.1556 & 0.0741 & 0.0947 & 0.2582 & 0.1163 \\
\hline & 6 & 0.0956 & 0.0966 & 0.0844 & 0.0841 & 0.2660 & 0.1103 & 0.0623 & 0.0959 & 0.3722 & 0.1236 \\
\hline & 7 & 0.1015 & 0.0963 & 0.0844 & 0.0842 & 0.3130 & 0.1156 & 0.0615 & 0.1038 & 0.2348 & 0.1263 \\
\hline & 8 & 0.1215 & 0.097 & 0.0844 & 0.0841 & 0.1058 & 0.2071 & 0.0499 & 0.1051 & 0.2777 & 0.1316 \\
\hline & 9 & 0.1051 & 0.1006 & 0.0844 & 0.0841 & 0.4266 & 0.1939 & 0.0632 & 0.1169 & 0.3793 & 0.1631 \\
\hline & 10 & 0.1158 & 0.0957 & 0.0844 & 0.0841 & 0.1773 & 0.3347 & 0.1027 & 0.1067 & 0.5806 & 0.1446 \\
\hline
\end{tabular}


hidden neuron and their connections, and finally aggregating the rules extracted at the individual levels from a composite rule set [20]. Therefore, after training the model, the network structure was analyzed and the activation value of each hidden neuron and the output neuron were calculated based on the inputs. This is done by Equation (3) and Equation (4).

$$
\begin{aligned}
& n e t=\sum_{i=1}^{n}\left(X_{i} W_{i}\right) b \\
& O=\text { TransferFunction(net) }
\end{aligned}
$$

Where in Equation (3), $n$ is the number of inputs of each neuron, $w_{i}$ is the connection weight and $x_{i}$ is the input value of each neuron, and $b$ is the bias of each hidden and output neuron. Then in Equation (4), transfer function was applied on the last value. Next, the activation values of hidden neurons obtained in the previous step were discrete in order to create the rules. To do this, the values greater than and equal to 0.5 were considered to 1 and the others 0 . Then for each hidden neuron, a truth table was formed whose inputs were the input values of database fed to the network. Then a logic function for each hidden neuron was calculated in terms of inputs. The same thing was done for the output neuron, but this time, discrete activation values of the hidden neurons were the inputs of the truth table. Finally, rules were generated by combining the two sets of rules and as a result the input mapping to output was provided in human-readable form of a set of rules. This method was tested for the Exclusive-OR (XOR) function with two, three and four bits and provided accurate results.

To illustrate more, the rule generation process for XOR function with two bits was explained. The arbitrary ANN structure consists of two input neurons, including $\mathrm{X}_{1}$ and $\mathrm{X}_{2}$ and three hidden neurons, including $\mathrm{H}_{1}, \mathrm{H}_{2}, \mathrm{H}_{3}$ and one output neuron and their biases.

The activation values of each hidden and output neuron were calculated according to the Equation (3) and Equation (4) and then were discrete. Then the truth tables were formed and logic function was calculated for each hidden neuron, separately. The results of this step are shown in Figure 2a. Then one the truth table was formed for output neuron in term of discrete activation hidden neurons. The results

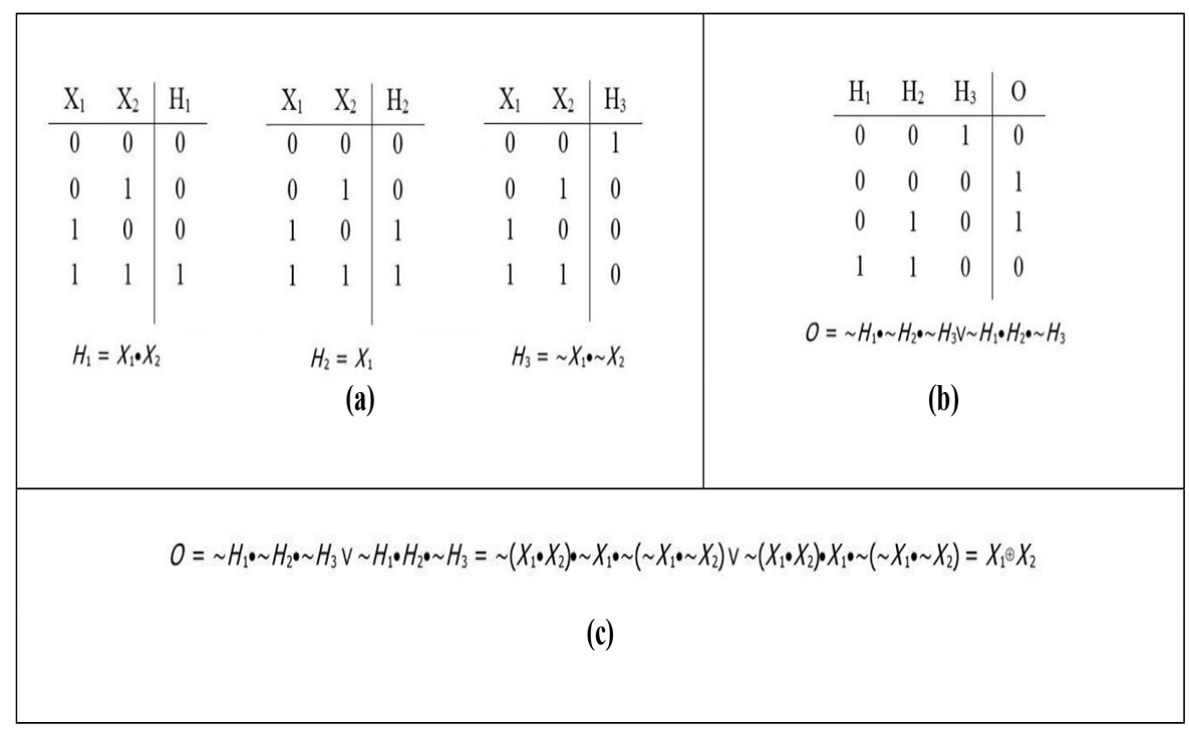

Figure 2: Generating rules for Exclusive-OR (XOR) Function. A: Calculating the logic function for each hidden neuron in term of input neurons; B: Calculating the logic function for output neuron in term of hidden neurons; C: Generating rules for output neuron in term of Artifcial Neural Networks (ANN's) input. 
of this step are shown in Figure 2b. Finally, all of obtained rules were combined. This combination is shown in Figure 2c.

On the other hand, the extracted rules for XOR function for two bits were interpreted as: If $\left(X_{1}=1\right.$ and $\left.X_{2}=0\right) \operatorname{Or}\left(X_{1}=0\right.$ And $\left.X_{2}=1\right)$ Then $O=1$; Else $O=0$.

\section{Pruning and generalization rules}

The extracted rules were completely specific. Therefore, generalization was made to cover the new training examples. After generating the rules, some of the rules that led to misclassification were pruned and then the rules of each class were generalized by the proposed algorithm in this paper as shown in Figure 3.

As shown in Figure 3, the proposed algorithm gets the set of extracted and pruned rules $R$ as inputs and $S$ is rule of each class which we have four of them. Then inconsistent values of conditions or attributes s of rules in $S$ become consistent or general, separately. To do this, each rule in $S$ named $d$ is checked. For each attribute $a_{i}$ in $S$, if $a_{i}$ is a binary attribute and is inconsistent with $a_{i}$ in $d, a_{i}$ will be removed from $S$. For example, gender, seizures in last six months and course of disease are binary and also if they have inconsistence values, they will be removed. For each attribute $a_{i}$ in $S$, if $a_{i}$ is a numeral attribute and greater or lesser than $a_{i}$ in $d$, the data range of $a_{i}$ in $S$ will be expanded. The numeral attributes are age, duration of epilepsy and hospitalization. For example, hospitalization has a variety of values for different instances in each class and after the generalization, the data range is expanded for example between 20 and 40 days. For each attribute $a_{i}$ in $S$, if $a_{i}$ is a categorical attribute, $a_{i}$ in $S$ will be replaced by some of the most frequent items. The categorical fields are made due to epilepsy, prior medication, status epilepticus type, etiology, seizure-controlling drugs. With generalization, some (at most three) values of the most frequent items for each class are extracted. For example, medication withdrawal and hypoxic are finding as the most frequent items for the etiology field.

\section{Integration of all systems}

To easily use the proposed method to prognosticate and identify the most important factors by physicians, an application was developed. In fact, this application uses the weights of the trained network and extracted rules.

\section{Results}

The obtained results in this study consist of two parts, including the results of the model

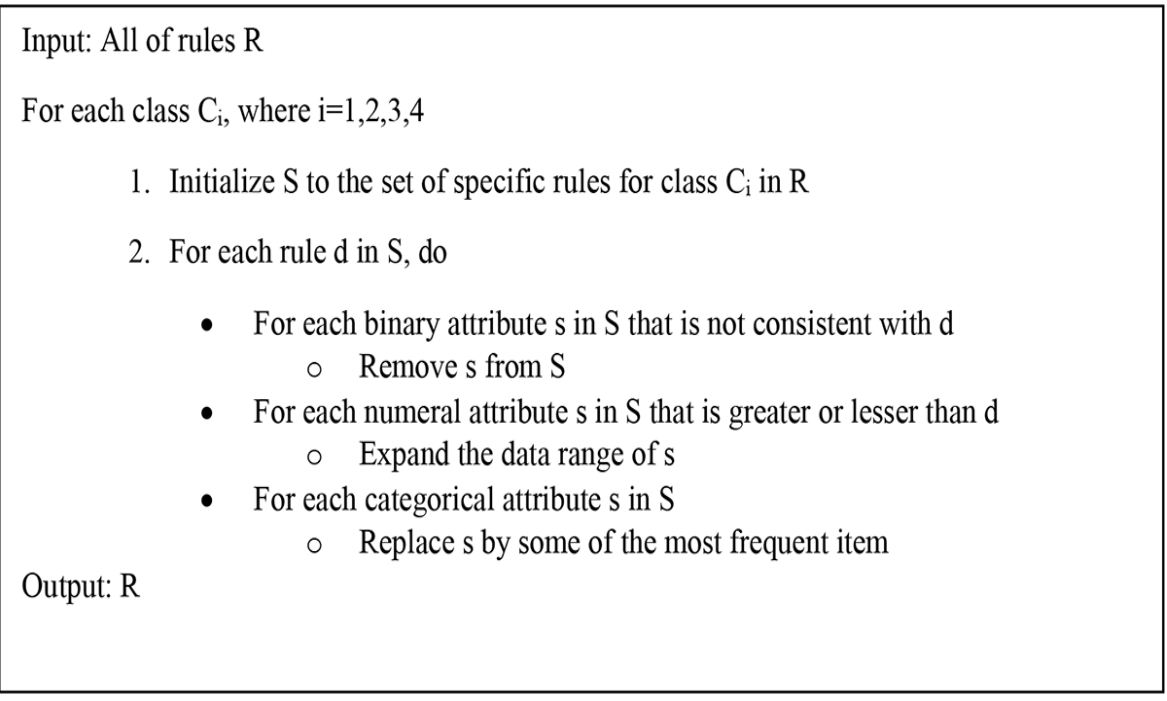

Figure 3: The proposed algorithm for rules generalization 
training in prognostication and the extracted rules which are described below.

The results of the model training in prognostication

Training automatically stopped when generalization ceased improving as indicated by an increase in MSE of validation samples. The results of applying the ANN methodology to distinguish between different outcomes of the patients with SE showed very good capability of the network to learn the patterns corresponding to symptoms of the patients with MSE 0.05. The network was simulated in the testing set (i.e. cases of the network has not seen before). The results were satisfied; the network was able to be classified by MSE 0.12 . Moreover, the best validation performance was 0.125 at epoch 237 as shown in Figure 4.

In Figure 4 the blue, red and green lines are representing a decrease MSE data for training, testing and validation data, respectively. The high proximity of the validation and testing lines is related to good data distribution and good training of the network [21]. To prove this, the confidence interval (CI) for validation and testing data was calculated by an accuracy of $99 \%$. In every line, there were 13 instances according to the distribution of data. We calculated the mean performance of trained sample (using the perform command in MATLAB), standard deviation and t-test due to the 13 instances. Then the confidence intervals were calculated with 99\% accuracy. The goal of this work is to investigate proximity of two lines at epoch 237, where the training is stopped. If the obtained CI for two lines overlap, it could be said that lines are not different, with $99 \%$ confidence and are quiet proximate. The calculation of the CI with t test was performed through Equation (5) and the calculations of the $\mathrm{CI}$ for the testing and validation lines are available in Equation (6) and Equation (7), respectively. Where in Equation (5) $\bar{X}$ is sample mean of data, $(s \div \sqrt{n})$ is standard deviation of data, $\alpha=0.01$ for $99 \%$ confidence-

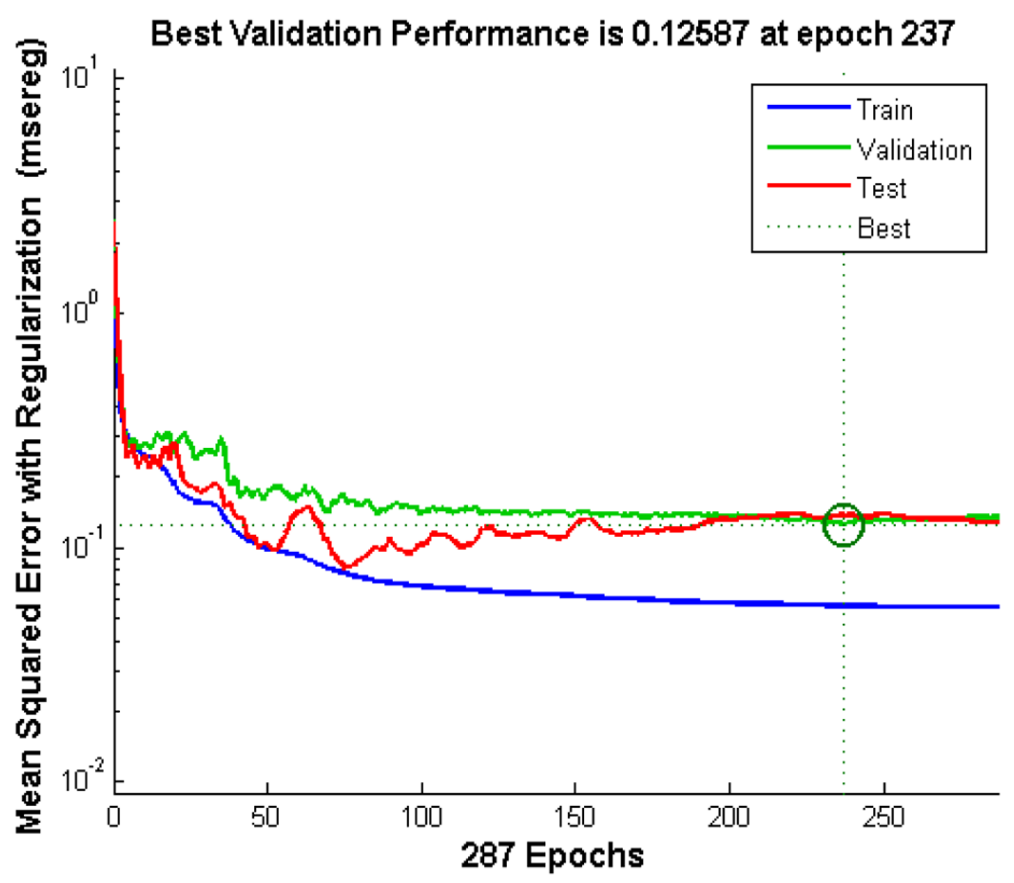

Figure 4: The best validation performance 
and $n=13$ is the count of samples.

Confidence Interval $=\bar{X} \pm t\left[1-\frac{\alpha}{2} ; n-1\right] \times(s \div \sqrt{n})$

TestSetCI $=0.1372 \pm 3.055 \times 0.2618=(-0.66260 .9370)$

ValidationSetCI $=0.1259 \pm 3.055 \times 0.1190=(-0.23760 .4894)$

According to the Equation (6) and Equation (7), the whole of validation CI is in testing $\mathrm{CI}$ and this is quite overlapping of two lines. Therefore, it could be said that lines are proximate and the model was trained well. In this study, for further analysis of the model, accuracy, precision and recall measures were used; they are available in Equation (8), Equation (9) and Equation (10).

Accuracr $=\frac{t p+t n}{t p+f p+f n+t n}=\frac{9}{13}=0.6923 \times 100 \% \cong 70 \%$

Precisn $=\frac{t p}{t p+f p}=\frac{\frac{3}{3}+\frac{1}{2}+\frac{3}{5}+\frac{2}{3}}{4}=0.6916 \times 100 \% \cong 70 \%(9)$

Recall $=\frac{t p}{t p+f n}=\frac{\frac{3}{5}+\frac{1}{1}+\frac{3}{4}+\frac{2}{3}}{4}=0.7541 \times 100 \% \cong 75 \%(10)$

Where in Equation (8), Equation (9) and Equation (10), tp, tn, $f p$ and $f n$ are true positive, true negative, false positive and false negative cases, respectively.

The results of the extracted rules

In this study, all feeding data on the ANN were converted to the rules as it was done for XOR function in section 2.3. Then the extracted rules were generalized by proposed algorithm in Figure 3 in 2.4 sections. All the gained outputs were in $[0,1]$ range. In order to produce understandable rules, all values were denormalized to the real and primary range by [1]. Then for nominal fields, the numeral values were converted to nominal values. Moreover, the rules were reformed in the form of If-then for easier understanding. These rules are available on the Figure 5a.

But, in Figure 5a can be seen some of the conditions have included all of the range and this are repeated in every four rules such as duration of epilepsy, cause of epilepsy, status type and hospitalization. Thus we can say that these conditions do not make differentiation between different groups and should be ignored. By removing these four conditions, the final obtained rules are shown in Figure $5 b$. In addition, in Figure 5b, the difference in the order of the values for the conditions in various rules cause to have the difference in their repetition rate such as prior-medication, etiology and seizures-controlling drug. For example, first group phenobarbital is more frequent than depakin and phenytoin as prior medication. However, different conditions don't have any priority in comparison with each other.

\section{Discussion}

As shown in Figure 5b, the first rule is about the patients with mortality GOS. They include all of the adult patients with SE whose previous treatments had been phenobarbital, depakin or phenytoin. Furthermore, the most important etiologies for this group were cerebral infarction, brain infection and hypoxia, respectively, and seizures management was possible with anesthetic drugs, phenytoin or depakin. It should be noted that the use of anesthetic drugs as seizure controlling drug is more common in this group than other groups. Anesthetic drugs are the most aggressive medications for status epilepticus and usually used for refractory kinds. Therefore, the more fatal underlying causes the more probable use of them.

The second rule is related to patients with severe disability GOS. They include all adults with phenytoin and carbamazepine as previous treatments. The most important etiologies of their seizures were cerebral infarction, brain infection and drug withdrawal, respectively; the seizure was controlled by phenytoin and anesthetic drugs. It is cclear that in this group, the use of phenytoin as seizure management is more than anesthetic drugs in comparison with 
IF $(20<=$ Age $<100)$ And $(0<=$ Duration $<=2)$ And (Cause=No_History or Secondary or Unknown) And (PriorMedication $=$ Phenobarbital or Depakine or Phenytoin) And (StatusType $=$ GTC) And (Etiology $=$ Cerebral Infarction or Brain Infection or Hypoxic) And (Seizure-controlling Drugs = Anesthetics or Phenytoin or Depakine) And $(2<=$ Hospitalization $<=100)$ Then GOS $=$ Mortality

IF $(20<=A g e<100)$ And $(0<=$ Duration $<=2)$ And (Cause= No_History or Secondary or Unknown) And (PriorMedication = Phenytoin or Carbamazepine) And (StatusType $=$ GTC) And (Etiology $=$ Cerebral Infarction or Brain Infection or Drugs Withdrawal) And (Seizure-controlling Drugs $=$ Phenytoin or Anesthetics) And $(2<=$ Hospitalization $<=100)$ Then GOS $=$ Severe Disability

IF $(20<=$ Age $<100)$ And $(0<=$ Duration $<=2)$ And (Cause $=$ No_History or Secondary or Unknown) And (PriorMedication $=$ Sodium Valproate or Carbamazepine) And (StatusType $=$ GTC) And $($ Etiology $=$ Drugs Withdrawal or Tumor or Metabolic) And (Seizure-controlling Drugs $=$ Phenytoin or Anesthetics or Phenobarbital) And $(2<=$ Hospitalization $<=100)$ Then GOS $=$ Moderate Disability

IF $(20<=$ Age $<60)$ And $(0<=$ Duration $<=2)$ And (Cause= No_History or Secondary or Unknown ) And (PriorMedication = Carbamazepine or Sodium Valproate) And (StatusType $=$ GTC) And (Course=0) And (Etiology $=$ Drugs Withdrawal or Drugs) And (Seizure-controlling Drugs $=$ Phenytoin ) And $(2<=$ Hospitalization $<=100)$ Then GOS = Good Recovery

(a)

IF (Prior-Medication $=$ Phenobarbital or Depakine or Phenytoin) And (Etiology $=$ Cerebral Infarction or Brain Infection or Hypoxia) And (Seizure-Controlling Drugs = Anesthetic drugs or Phenytoin or Depakine); Then GOS = Mortality

IF (Prior-Medication $=$ Phenytoin or Carbamazepine) And (Etiology $=$ Cerebral Infarction or Brain Infection or Drug Withdrawal) And (Seizure- Controlling Drugs = Phenytoin or Anesthetic drugs); Then GOS = Severe Disability

IF (Prior-Medication $=$ Sodium Valproate or Carbamazepine) And (Etiology $=$ Drug Withdrawal or Tumor or Metabolic) And (Seizure- controlling drugs $=$ Phenytoin or Anesthetic drugs or Phenobarbital); Then GOS = Moderate Disability

IF $(20 \leq$ Age $<60)$ And (Prior-Medication = Carbamazepine or Sodium Valproate) And $($ Course $=0)$ And (Etiology $=$ Drug Withdrawal or Drug/Substance abuse) And (Seizure- Controlling Drugs = Phenytoin ) Then; GOS = Good Recovery

Figure 5: The Result of the extracted rule set from the model. a: Primary extracted rules; b: Final extracted rules.

the prior group.

The third rule is about patients with moderate disability GOS. However, they also consist of all adult patients, this time previous treatments include sodium valproate and carbamazepine. The most important etiologies for this group were antiepileptic drugs withdrawal, tumor and metabolic problems, respectively. Seizure management of this group was possible through Phenytoin, anesthetic drugs and phenobarbital.

Finally, the fourth rule is for patients with good recovery GOS. They comprise those adults younger than 60 years with sodium val- 
proate and carbamazepine as previous treatments. The most important etiologies of seizure were antiepileptic drugs withdrawal and drug/substance abuse and their seizures were only controlled by phenytoin.

According to the description, it can be concluded that there is less probability for younger patients to die and they usually recover. Previous treatments for people with high brain injuries (i.e. groups 1 and 2), were mostly phenobarbital, depakin and phenytoin, and the other two groups who suffered less injuries had used sodium valproate and carbamazepine and also cerebral infarction (stroke) was identified as the main etiology for the first and second groups; thus it is considered as a poor prognosis for those patients. Furthermore, drugs withdrawal and drug/substance abuse were identified as the main etiologies for the third and fourth groups, and it is a good prognosis for them. Since drug factors are preventable with practical education and awareness of the patients with SE, thus they can prevent from brain injuries.

It is noticeable that anesthetic drugs were mostly used to control seizures for people who died, and with decrease of the brain injuries, taking these drugs decreased. Therefore, for those who recovered, the use of these drugs is minimized. The relationship between age and outcome has been identified previously, and cerebral infarction (stroke) and drugs withdrawal were introduced as the main etiology (Poursadeghfard et al., 2014) [10]. Above all, older patients had a higher risk of death in comparison with younger ones, and stroke and tumor were poor prognosis (Rossetti et al., 2006) [13]; however, Poursadeghfard et al. (2014) and Rossetti et al. (2006) did not say anything about prior medication or seizer-controlling drugs.

Thus, the ANN method could be classified and predict with about $70 \%$ accuracy. Although $70 \%$ accuracy may not be acceptable in the medical field, due to the nature of the problem and the shortage of data and also based on the results obtained from implementation other data mining algorithms such as Bayesian network and decision tree, i.e. random forest, which have great abilities to predict and generate rules, it could be argued that the proposed method has provided higher accuracy. The results of implementation of Bayesian network and random forest methods, on same data and 10 -fold cross validation in Weka software, are shown in Table 3. In the random forest method, the maximum depth of the trees was infinitive, and the number of trees generated was 10 and the random number seed used was 1 and, in the Bayesian network, simple estimator was used for estimating the conditional probability tables of bayes network and also hill climbing algorithm was used for searching algorithm.

Therefore, according to the results of other data mining techniques in Table 3 , we could say the ANN approach is better; but it is likely that the combination of ANN with the cat swarms optimization (Yusiong, 2012) [22]. Algorithm will provide better results and it must be tested in the future works.

Table 3: Performance evaluation of some methods of data mining

\begin{tabular}{cccc} 
Method & $\begin{array}{c}\text { Accuracy } \\
\%\end{array}$ & $\begin{array}{c}\text { Precision } \\
\%\end{array}$ & $\begin{array}{c}\text { Recall } \\
\%\end{array}$ \\
\hline ANN & 70 & 70 & 75 \\
\hline Bayesian Network & 51 & 50 & 50 \\
\hline Random Forest & 46 & 45 & 45
\end{tabular}

ANN: Artifcial Neural Network

\section{Conclusion}

In this study, an application was proposed to manage the SE patients' data, and determine the prognosis of their outcome on discharge via an intelligent rule-based method, and also identify the most important influential factors. As noted previously, intelligent approaches about the SE included the analysis of EEG signals tests and there are not any intelligent methods based on the symptoms of the SE. 
An Intelligent Rule-based System

The proposed method was able to predict the outcome of patients with SE on discharge which is accurately close to $70 \%$. Therefore, if the prognosis was poor, treatment would be more sensitive. In addition, the course of the disease, prior medication, seizure-controlling drugs and other etiology values were identified as important factors besides age, medication withdrawal and cerebral infarction (stroke) introduced finally, the ANN approach could provide higher accuracy than other methods of data mining.

\section{Acknowledgment}

The authors would like to thank Dr. Nasrin Shokrpour at Center for Development of Clinical Research of Nemazee Hospital for editorial assistance.

\section{Conflict of Interest}

None

\section{References}

1. Lowenstein DH, Alldredge BK. Status epilepticus. N Engl J Med. 1998;338:970-6. doi: 10.1056/ NEJM199804023381407. PubMed PMID: 9521986

2. Watson C. Status epilepticus. Clinical features, pathophysiology, and treatment. West J Med. 1991;155:626-31. PubMed PMID: 1812633. PubMed PMCID: PMC1003112.

3. Moayedi A, Atashabparvar A, Eftekhari E. Status epilepticus: etiology, outcome and predictors of mortality. Iranian Journal of Child Neurology. 2007;2:19-23.

4. Riviello Jr JJ, Ashwal S, Hirtz D, Glauser T, BallabanGil K, Kelley K, et al. Practice parameter: diagnostic assessment of the child with status epilepticus (an evidence-based review): report of the Quality Standards Subcommittee of the American Academy of Neurology and the Practice Committee of the Child Neurology Society. Neurology. 2006;67:154250. doi: 10.1212/01.wnl.0000243197.05519.3d PubMed PMID: 17101884.

5. Jackson J. Data mining; a conceptual overview. Communications of the Association for Information Systems. 2002;8:19.

6. Kamruzzaman S, Islam M. An algorithm to extract rules from artificial neural networks for medical di- agnosis problems. arXiv preprint arXiv:10094566. $2010 ; 12(8): 41-59$.

7. 7. Farrokhi A, Talebi N, Safari F, editors. Automatic detection of epileptic spike using fuzzy ARTMAP neural network. Proceedings of the 10th WSEAS international conference on Signal processing, computational geometry and artificial vision; Taipei, Taiwan: World Scientific and Engineering Academy and Society (WSEAS); 2010.

8. Sukanesh R, Harikumar R. A Comparison of Genetic Algorithm \& Neural Network (MLP) In Patient Specific Classification of Epilepsy Risk Levels from EEG Signals. Engineering Letters. 2007;14(1).

9. Aslan K, Bozdemir H, Sahin C, Noyan Ogulata S. Can neural network able to estimate the prognosis of epilepsy patients according to risk factors? J Med Syst. 2010;34:541-50. doi: 10.1007/s10916009-9267-8. PubMed PMID: 20703908.

10. Poursadeghfard M, Hashemzehi Z, Ashjazadeh N. Status Epilepticus in Adults: A 6-Year Retrospective Study. Galen Medical Journal. 2014;3:153-59.

11. Holtkamp M, Othman J, Buchheim K, Meierkord $\mathrm{H}$. Predictors and prognosis of refractory status epilepticus treated in a neurological intensive care unit. J Neurol Neurosurg Psychiatry. 2005;76:5349. doi: 10.1136/jnnp.2004.041947.

12. Kwong KL, Chang K, Lam SY. Features predicting adverse outcomes of status epilepticus in childhood. Hong Kong Med J. 2004:10:156-9. PubMed PMID: 15181218.

13. Rossetti A, Hurwitz S, Logroscino G, Bromfield E. Prognosis of status epilepticus: role of aetiology, age, and consciousness impairment at presentation. J Neurol Neurosurg Psychiatry. 2006;77:6115. doi: 10.1136/jnnp.2005.080887.

14. Adibeik B. Status epilepticus: a review. Iranian Journal of Child Neurology. 2008;2:7-14.

15. Akhondian J, Heydarian F, Jafari SA. Predictive factors of pediatric intractable seizures. Arch Iran Med. 2006;9:236-9. PubMed PMID: 16859058.

16. Ashrafi MR. Status Epilepticus. Iranian Journal of Pediatrics. 2000;10:204-17.

17. Moayedi A, Atashabparvar A, Eftekhari E. Status epilepticus: etiology, outcome and predictors of mortality. Iranian Journal of Child Neurology. 2007;2:19-23

18. Tabatabaei SS, Delbari A, Salman-Roghani R, Shahgholi L, Fadayevatan R, Mokhber N, et al. Seizures and epilepsy in elderly patients of an urban area of Iran: clinical manifestation, differential diagnosis, etiology, and epilepsy subtypes. Neurol 
Sci. 2013;34:1441-6. doi: 10.1007/s10072-0121261-0. PubMed PMID: 23232961.

19. Jennett B, Bond M. Assessment of outcome after severe brain damage. Lancet. 1975;1:480-4. PubMed PMID: 46957.

20. Huysmans J, Baesens B, Vanthienen J. Using rule extraction to improve the comprehensibility of predictive models. Behavioral \& Experimental Economics. 2006:1-55. doi: 10.2139/ssrn.961358.
21. Biswas SK, Mia MMA. Image Reconstruction Using Multi Layer Perceptron (MLP) And Support Vector Machine (SVM) Classifier And Study Of Classification Accuracy. International Journal of Scientific \& Technology Research. 2015;4:226-31.

22. Yusiong JPT. Optimizing artificial neural networks using cat swarm optimization algorithm. International Journal of Intelligent Systems and Applications. 2012;5:69-80. 